\title{
THE ONLINE SYSTEM FOR LIDAR DATA HANDLING AND REAL TIME MONITORING OF LIDAR OPERATIONS AT ALO-USU
}

\author{
Luis A. Navarro ${ }^{1 *}$, Vincent B. Wickwar ${ }^{1}$, Jose Gamboa ${ }^{2}$, Marco Milla ${ }^{2}$ \\ ${ }^{1}$ Physics and Center for Atmospheric and Space Science, Utah State University, Logan, UT 84322, USA, \\ *Email: luis.navarro.dominguez@gmail.com \\ ${ }^{2}$ Jicamarca Radio Observatory, Instituto Geofisico del Peru, Lima, Peru
}

\begin{abstract}
It is no longer sufficient to use lidar, such as the Rayleigh lidar at the Atmospheric Lidar Observatory (ALO) at Utah State University (USU), to observe the middle atmosphere and reduce the data to geophysical parameters. Extended operations, with inevitable equipment, data reduction, and analysis improvements, require us to keep careful track of all these changes and how they affect the scientific products. Furthermore, many of the funding agencies and the journals now require us to do, at least, some of this. We have built three interconnected data structures to organize and manage the different hardware and software setups from the Internet as well as to keep track of the products generated by these. These structures were implemented as a database, which is particularly important for groups with a large volume of information like the Rayleigh Group at ALO-USU.
\end{abstract}

\section{INTRODUCTION}

The Rayleigh-scatter Lidar Group in the Center for Atmospheric and Space Sciences (CASS) at Utah State University has been developing and operating Rayleigh lidars since 1992 to study the middle atmosphere. During this period, different hardware and software have been developed and implemented to improve the lidar's capabilities and the scientific products. More generally, researchers in many fields have faced a similar challenge keeping track of the constantly improving hardware and software in their systems and their effects. To help, they use several tools such as Evernote, TeamViewer, Google Drive, as well as the traditional logbooks and notebooks. However, few of these tools have been designed for scientific instrumentation, let alone for lidar systems.

In addition, to make it easier to verify scientific conclusions and to make scientific results more available to tax payers, funding agencies such as NSF and NIH have added a requirement for proposals to include a Data Management Plan. These plans are focused, in general, on the management, preservation and sharing of data and, more specifically, on sharing the scientific products generated by the proposed research.

So far, different institutions and communities have developed their own solutions for sharing and presenting results. Among others, this is the case of the DMPTool [1] developed by the University of California, San Diego. This is an online platform that helps produce the required Data Management Plan document. This platform uses different document templates, depending on the funding agency, and lets one institution share them with other institutions doing similar research. This last characteristic is particularly important for promoting networking and collaboration among groups.

Another example is the OpenMadrigal Initiative [2]. It is a platform, developed in Python that supports an on-line database for atmospheric data produced by a variety of instruments and models. It enables anyone to examine and download the results of scientific measurements from the web. It includes some lidar data. Contents of the database can be downloaded in several possible formats, such as the CEDAR and HDF5 formats.

Aware of this necessity, the Optical Instrumentation Department from the Jicamarca Radio Observatory started a development for optical data handling that was subsequently specialized and improved for lidar instrumentation at USU.

In the following section, a description of the data structures for the Rayleigh Group at ALO-USU is presented. Section 3 shows some of the online features available from the Internet. Finally, Section 4 indicates how it is being used at USU for lidar and correlative data. 


\section{METHODOLOGY}

The system is based on three different structures designed to save different types of information. These are interconnected to give the user the ability to save and retrieve information in a quick and ordered way.

\subsection{Structure for the Instrumentation}

This structure is based on past and present experience operating optical instrumentation. It systematically organizes the relationships between the instrumentation and the observations

It starts by defining a Type of Instrument, which in turn is used to define many Instruments, each of which are mainly described by a Type, Geographical Location and a Person to Contact. It also has a Code to match with other classifications such as the instrument code in the Madrigal data model [2]. For each instrument, we can define several Instrument Models, which are meant to describe the instrument hardware through a set of parameters and their values. The Rayleigh Group at ALO-USU used a design from 1992 to 2004, called "Old design". Since 2012, it has used another design, called "New Design". Many of these terms, and examples, are shown in Figure 1.

Following up, several Operating modes might be available due to the hardware specifications for each Instrument Model. Each Operating Mode describes how the hardware parameters interact through a set of software settings that control how the hardware functions.

As an example, the Instrument Model "New Design" has servomotors to position the optical fiber at the focal point of the telescope mirror [4]. This is an Operating Mode called "Calibration". In addition, this Instrument Model is able to collect data continuously throughout the night in an Operating Mode called "Extended Sounding". This is possible through the Value of Setting for the number of laser pulses to integrate over [4]. These can also be seen in Figure 1.

In the same sense, due to the Value of Settings, each Operating Mode opens up the capability of performing different experiments. An Experiment can also be reached by many Operating Modes. Then, an experiment Trial represents the execution of the Experiment for a specified date or time interval. In Figure 1, the experiment Coupling Temperatures could measure temperatures and relative densities from the stratosphere to the lower thermosphere.

In addition, Trials are related to a Campaign, which is a label given to an interval of time that usually characterizes the motivation or purpose of an Experiment or set of observations. This is particularly important when different groups run different instruments. Examples are described for a series of optical campaigns (IMPAC) [3].

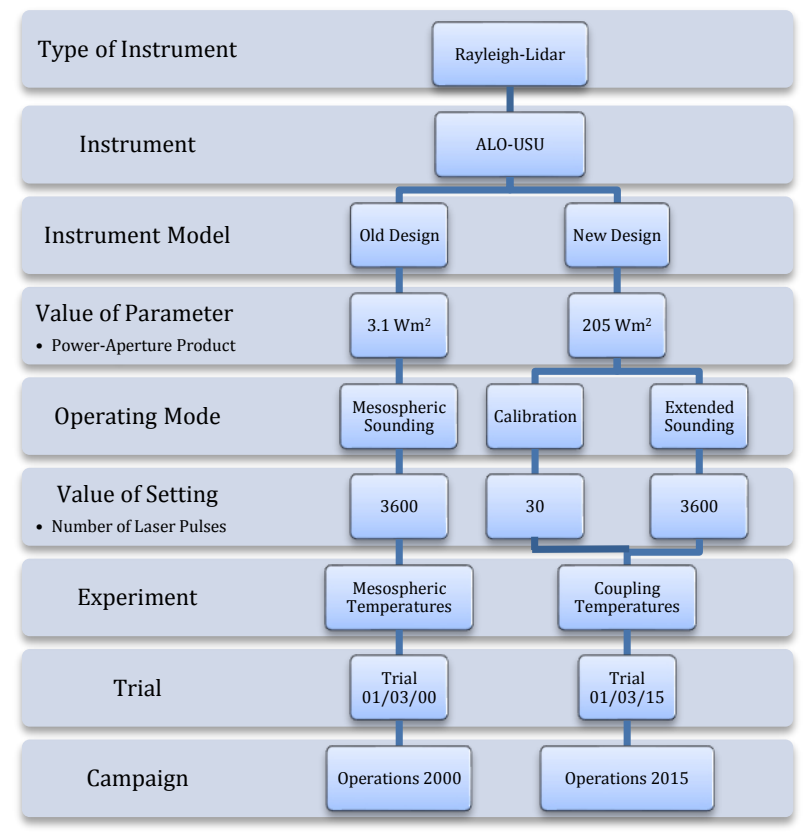

Figure 1. Configuration of the instrumentation structure for Type of Instrumemt, Rayleigh Lidar, at USU.

\subsection{Structure for Products}

This structure is intended to manage the Type of Product Set. A Product is, in general, any type of information generated by a Trial of an Experiment or by a Campaign such as files or data depending on what information is required to be handed directly from the database. These products can be classified in Types of Product Sets like raw data, processed data, published data or any other type defined by the user. Also, even when the Generators of Products are meant to be a Trial or a Campaign, other types of Generators can be setup by the user.

For ALO-USU, four different Types of Product Sets are defined: "Calibration Data", "Raw Data", "Temperature Data" and "Cedar Data". The 
"Raw Data", shown in Figure 2, is designed for the data recorded from a Multi-Channel Scaler in normal operation, whereas the Type of Product Set "Calibration Data" is used for data recorded during telescope alignment. In the same sense, the Type of Product Set "Temperature Data" is for data generated by software that processes the Raw Data to produce mesospheric temperatures. "Cedar Data" is used for the processed data that is transformed into the Cedar data format.

On the other hand, the user can process a Product Set to generate more information. Usually, the routines, scripts or programs that process information need initial conditions or, in general, specific setups that the software will use to perform the task.

For the case of Raw Data generated by the experiment "Coupling Temperatures", a task was created to reduce these data and to save the resulting temperatures in the database as products with type "Temperature Data". To fulfill this task, to determine the temperatures, we need an initial temperature [6], which was setup as a condition.

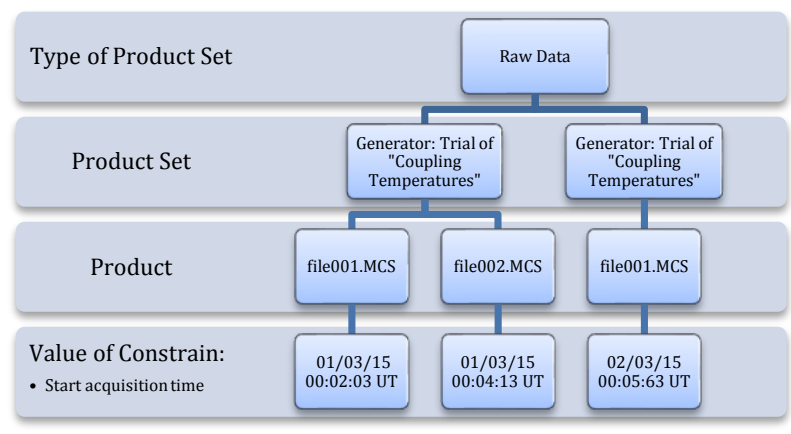

Figure 2. Configuration for Type of Product Set "Raw data" for ALO-USU.

\subsection{Structure for Supervisory Devices}

To supervise the operation of the lidar system, we define an entity called the Supervisory Device (SD). It is a web server running on the computer controlling the lidar operation. It receives and executes commands to inform the lidar operator about the lidar status or to send back data files to be saved.

A basic set of commands were implemented in the SD so as to perform an initial registration of the SD (see Figure 3), list directories, send files, start a watchdog thread to look over any newly generated file and send it to the server, start a ping thread to find the average communication time to check the network connection quality (e.g., Figure 4), send a status command and send a shutdown command to the SD.

For the instrument Rayleigh lidar at ALO-USU, this was implemented with the HTTPServer class of the Python core library. The results, state and variables handled by this communication are saved in the database following this structure.

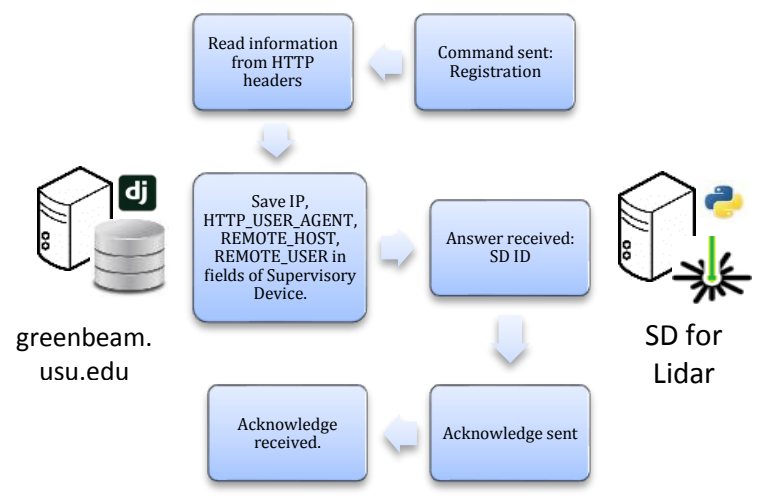

Figure 3. Procedure for registering the SD into the Supervisory Device structure.

\section{RESULTS}

For the Rayleigh Lidar Group at ALO-USU, the site https://greenbeam.usu.edu was implemented to monitor and handle the lidar data (see Figure 5). The database engine MySQL and the Python web framework Django are used to implement the structures discussed in Section 2. The scheduling of tasks is done by the distributed task queue system Celery. The Google API is used to handle user authentication and the data are backed up into the cloud storage Box.

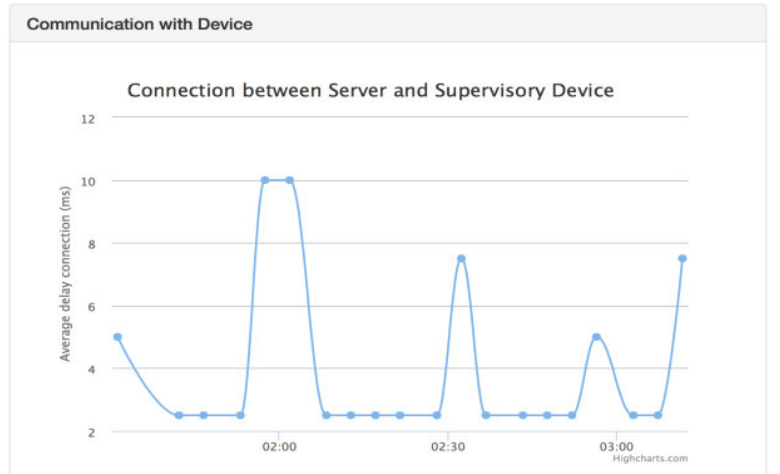

Figure 4. Example of online chart showing the quality of the connection to the Supervisory Device. 


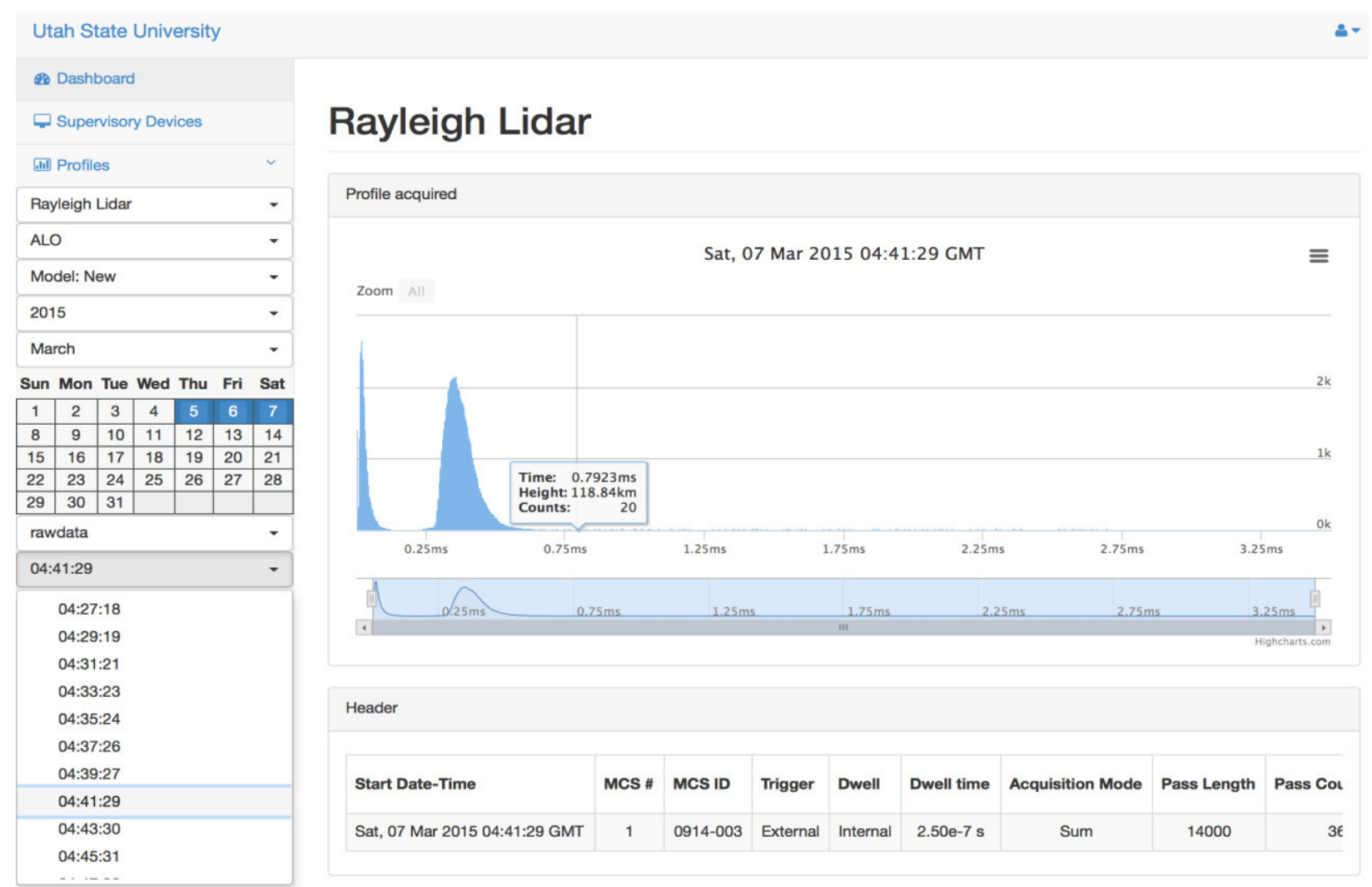

Figure 5. Example of online viewer of Products for the Rayleigh Lidar at ALO-USU.

\section{CONCLUSIONS}

The interconnected design of the structures and the use of informatic tools like MySQL, Django, and Celery (see Figure 6), make it possible to handle a large volume of data. While this was developed for lidar at ALO-USU, much of this can be adapted to the needs of other lidar groups.

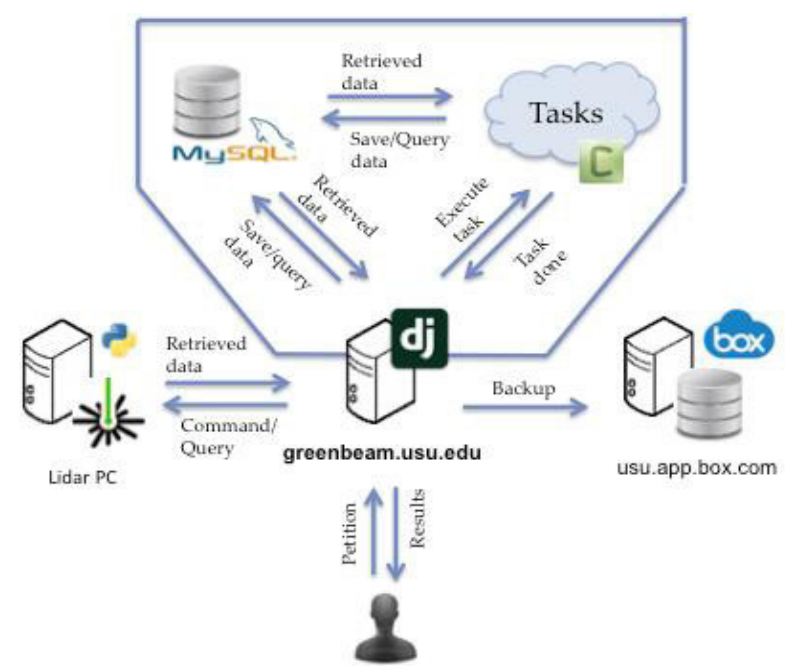

Figure 6. Data flow design for the Rayleigh Lidar at ALO-USU located in https://greenbeam.usu.edu.

\section{REFERENCES}

[1] https://dmptool.org

[2] http://www.openmadrigal.org

[3] Wu, Q., W. Wang, M. G. Conde, A. L. Arulian, R. Niciejewski, and J. W. Meriwether, 2009: Ionosphere Mesosphere Polar Aeronomy Campaign (IMPAC). AGU Fall Meeting 2009, San Francisco, CA.

[4] Wickwar, V. B., L. Sox, D. L. Barton, J. P. Herron, J., and M. T. Emerick. June 22, 2014: Extremely Sensitive Rayleigh-Scatter Lidar at USU. Presented at the 2014 CEDAR Workshop, University of Washington, Seattle, WA.

[5] Peterson, L. W., 2011: Lidar User's Manual, Undergraduate Honors Theses, 20 pp, Utah State University, Logan, UT.

[6] Wickwar, V. B., and L. Sox, June 22, 2014: Interpretation (or is it Calibration?) of RayleighScatter Lidar Signals, CEDAR Workshop, University of Washington, Seattle, WA. 